\title{
Thrust Vector Control Robustness of axial-symmetric Launch Vehicles with Fuel Slosh
}

\author{
Tiziano Pulecchi and Andres Marcos \\ DEIMOS SPACE S.L.U., Ronda de Poniente 19, 28760, Madrid,, Spain \\ Christophe Roux and Valerio Mantini \\ ELV, Corso Garibaldi 22, 00034, Colleferro (Rome), Italy \\ Samir Bennani \\ ESA/ESTEC, Keplerlaan 1, 2201 AZ, Noordwijk, The Netherlands
}

\begin{abstract}
Clearance of Thrust Vector Control (TVC) laws for launchers demands the capability to provide analytical certificates for the system's robust stability. During exo-atmospheric flight, a key role is played by the ability to deal with non rigid-body effects such as fuel sloshing. Depending on its frequency and magnitude, sloshing can easily deteriorate the performance of the TVC laws to the point of threatening the stability of the system. While the current Verification and Validation ( $\mathrm{V} \& \mathrm{~V})$ approach used in industry is limited inasmuch as it entails assessment of control law robustness using a finite set of conditions, advanced analysis techniques have the potential to clear the entire analysis space. In this paper the structured singular value analysis is used to obtain robust stability certificates for the attitude motion of an axial-symmetric launch vehicle (LV) subject to fuel sloshing.
\end{abstract}

\section{Nomenclature}

$\beta$
$\beta_{c}$
$\zeta$
$\zeta_{a c t}$
$\theta$
$\lambda$
$\omega$
$\omega_{a c t}$
$k$
$k_{2}, k_{4}$
$s$
$j$
$j_{p l}$
$J$
$m$
$m_{p l}$
$M$
$M_{s l o s h}$
$T$
$x_{c g}$
$x_{c g, p l}$
$x_{C G}$
$x_{C G, s l o s h}$
$x_{P V P}$
$y$

$=$ TVC deflection angle

$=$ (commanded) TVC deflection angle

$=$ damping ratio

$=$ damping ratio of engine's nozzle dynamics

= yaw attitude

$=$ payload's cylindrical tank height-over-diameter ratio

$=$ natural frequency

$=$ natural frequency of engine's nozzle dynamics

$=$ elastic constant of sloshing model

$=$ Tail-Wags-Dog effect coefficients

$=$ position of the sloshing fuel's center of mass along the $\mathrm{y}$ direction

$=$ moment of inertia of LV without payload

$=$ moment of inertia of payload

$=$ moment of inertia of overall LV

$=$ mass of LV without payload

$=$ mass of payload

$=$ mass of overall LV

$=$ sloshing mass

$=$ rocket engine's thrust

$=$ center of gravity position along the centerline of $\mathrm{LV}$ without payload

$=$ center of gravity position along the centerline of payload

$=$ center of gravity position along the centerline of overall LV

$=$ center of gravity position along the centerline of sloshing mass

$=$ engine nozzle's pivot point position along the centerline

$=$ lateral displacement 


\section{Introduction}

CLEARANCE of Thrust Vector Control (TVC) laws for launchers demands the capability to provide analytical certificates for the system's robust stability. The current state-of-practice for launcher Verification \& Validation $(\mathrm{V} \& \mathrm{~V})$ combines analysis of probabilistic time domain requirements through nonlinear simulations as well as analysis of frequency domain requirements for a finite set of conditions. Although very practical, this approach has many disadvantages in that it relies on massive amounts of computations without guaranteed proofs on the full parameter space. To overcome these limitations many advances have been explored in the field of aeronautical and military GNC V\&V based on advanced theories and tools ${ }^{1,2}$. Among them, use of $\mu$ analysis ${ }^{3-7}$ is especially appealing because of its potential to allow clearance of the complete analysis space in a computationally efficient manner.

One key-factor the analyst must tackle during the V\&V of a complex dynamical system such as a launch vehicle consists in certifying the robustness of the control laws in presence of flexible dynamics and against fuel sloshing induced by non-coaxial accelerations. Because of the inherent complexity and relevancy of the subject, in this paper we focus on studying the effect of fuel sloshing alone, while at the same time making the common assumption that our case-study launch vehicle $(\mathrm{LV})$ is rigid enough that elastic modes have second order effect on stability margins.

Controlling liquid fuel sloshing within a launch vehicle is a major design concern. A commonly adopted solution to suppress fuel sloshing inside a launch vehicle is by adoption of physical barriers, such as baffles, meant to limit the movement of liquid fuel inside its containers. Slosh-suppression, however, is generally limited to the vehicle itself, while it is seldom addressed as part of the payload's design cycle. In today's large and complex spacecraft, a substantial mass of fuel is allocated to allow performing attitude and orbital control tasks such as orbit insertion, orbital maneuvers, detumbling, station-keeping, etc. As a result, the fuel over spacecraft mass ratio can reach significant figures (e.g., in the case of geosynchronous satellites) with the fuel amounting to approximately $40 \%$ of the total mass of the spacecraft.

With respect to the use of mu-analysis, a major step prior to the analysis is the derivation of the linear fractional transformation (LFT) model to be used for analysis ${ }^{4,6}$. An LFT is the representation of a feedback system described as the feedback interconnection of two causal and bounded mathematical operators: a feed-forward operator $M$ and a feedback operator $\Delta$ used to describe the model uncertainties, nonlinearities and time variability (see Fig. 2). Within this framework, systems are regarded as operators mapping a normed vector space into another, whereas the input/output signals belong to these vector spaces.

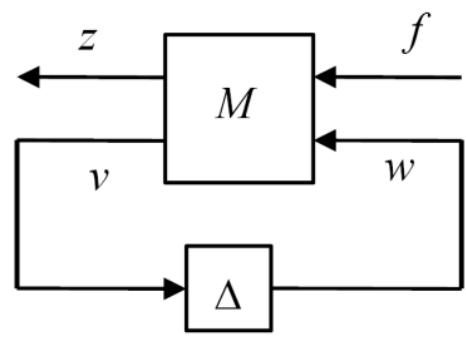

\section{Figure 2: Typical feedback interconnection of an LFR model.}

This LFT modeling step represents always a critical point as this type of models increase in dimension as more parameters and complex dependencies are included quickly reaching a point where the current mu-analysis algorithms break down. Thus, it is necessary to perform a trade-off between the fidelity of the model (to the nonlinear knowledge of the system) and the reliability of the analysis. In other words, a simpler model capturing relevant dependencies might provide satisfactory, albeit probably partial, analysis results otherwise not possible with a higher-fidelity model. Dimension versus fidelity of the LFT model is thus an especially critical trade-off for mu analysis.

In this article, we analyze the robust stability of a traditional PID Thrust Vector Control law for a launch vehicle with fuel sloshing and during exo-atmospheric flight. Cylindrical fuel tanks are considered, and the complex sloshing dynamics is approximated with its lowest frequency slosh mode using a spring-mass analogy. For simplicity's sake, and because we are analyzing an exo-atmospheric phase of flight, both aerodynamic effects and elastic modes are ignored. The TVC control objective is to track a reference trajectory in space by suitably orienting the rocket engine's nozzle relative to the launch vehicle itself. Our goal consists in providing a methodology to certify that the TVC system specification requirements are met in presence of fuel sloshing. The outline of this paper is as follows. In Section 2 an overview of the problem is given. Section 3 presents the analysis results for the full payload case, while Section 4 presents those for the single payload case. Finally, conclusions are drawn in Section 5. 


\section{Launch Vehicle Dynamics}

\section{A. Open-loop Plant}

TVC control is used to track a reference (optimal) trajectory in space by suitably controlling the orientation of the rocket engine's nozzle relative to the LV itself. For low roll rates and because of the vehicle axial symmetry, the pitch, yaw and roll motions are virtually decoupled. Furthermore, the pitch/yaw TVC control laws are identical as well. Proving robust stability for the pitch channel is then equivalent to prove robust stability for the yaw channel, and vice versa. Based on this assurance, the analysis of the yaw motion alone is addressed.

In this framework, the (yaw) rigid body motion is planar and completely described by its yaw attitude $\theta$ and linear motion $y$ (taking place in a direction transversal to the projection of the velocity vector in the pitch/roll plane) in a frame linked to the velocity of the reference trajectory, see Figure 1.

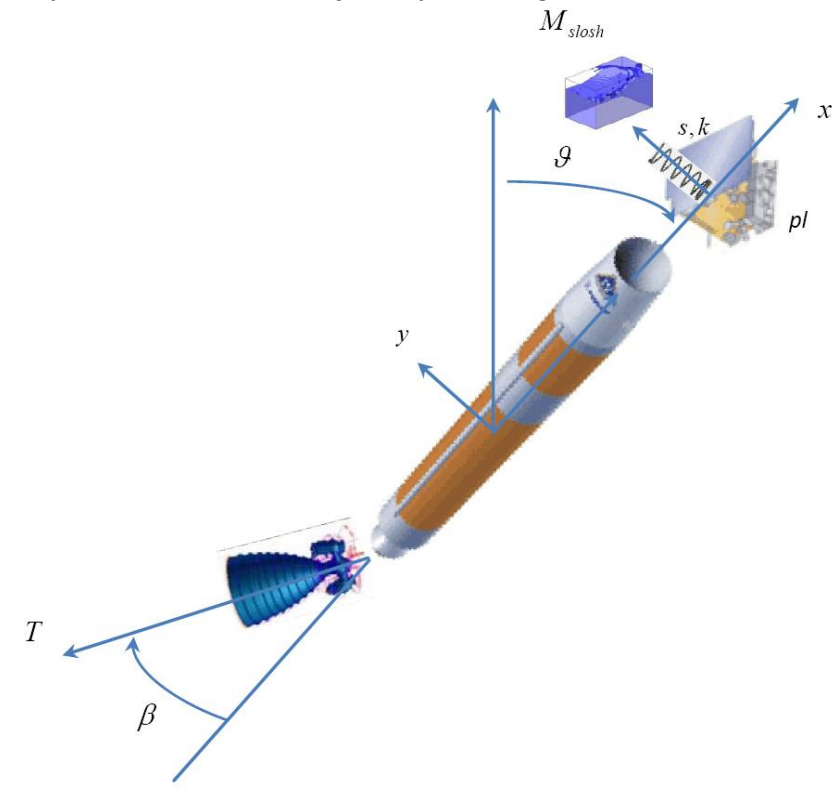

Figure 1 Single axis TVC control problem schematic, including payload fuel tanks' sloshing.

Transversal accelerations (in the $y$ direction) cause the fuel stocked in the payload's tanks to slosh. Depending on its frequency and magnitude, this motion can significantly deteriorate the system's performance, to the point of threatening to destabilize the system. Note that only sloshing due to payload needs be considered, because the rocket engines' fuel tanks are commonly equipped with suitable damping devices that effectively inhibit fuel sloshing.

Several sloshing models were proposed in the literature ${ }^{8}$. Among these, the most prominent are the spring-like and pendulum-like sloshing models. Such models describe the complex sloshing dynamics with a single mode representation where the whole sloshing mass is assumed to rigidly move, as if "frozen", in its tank. In this paper, a spring-like sloshing model is adopted, where the sloshing motion envisions a rigid motion of the fuel in the $y$ direction (fuel slosh responding to cross accelerations). The effect of the sloshing mass on the system is then modeled as an elastic force responding to the fuel displacement in the y direction.

Let $s$ denote the position of the sloshing fuel's center of mass along the $y$ direction. Furthermore, let the only force acting on the system be the engine's thrust $T$. (LV aerodynamics as well as servo-elastic couplings can indeed be considered as second order effects during exo-atmospheric flight.) Finally, let $\beta$ and $\beta_{c}$ be the Thrust Vector Control (TVC) actual and commanded deflection angles. Then the system dynamics, including fuel sloshing and Tail Wags Dog (TWD) effect, due to inertia of the engine's nozzle, are fully defined by the following four equations describing respectively, the LV's translational and angular motion, the sloshing motion and the nozzle dynamic:

$$
\begin{aligned}
& M \ddot{y}=-T \beta+k s+k_{2} \ddot{\beta} \\
& J \ddot{\vartheta}=T \beta\left(x_{P V P}-x_{C G}\right)+\left(x_{C G, s l o s h}-x_{C G}\right) k s+k_{4} \ddot{\beta} \\
& \ddot{y}+\left(x_{C G, s l o s h}-x_{C G}\right) \ddot{\vartheta}+\ddot{s}=-2 \zeta \omega \dot{s}-\omega^{2} s \\
& \ddot{\beta}+2 \zeta_{a c t} \omega_{a c t} \dot{\beta}+\omega_{a c t}^{2} \beta=\omega_{a c t}^{2} \beta_{c}
\end{aligned}
$$


The system's equations depend parametrically on a set of seven independent parameters that are referred to as derived parameters. These are: the mass $M$, moment of inertia $J$ and center of gravity position along the centerline $x_{C G}$ for overall LV (including its payload), center of gravity position of the sloshing mass $x_{C G \text {,slosh }}$ "elastic" constant $k=\omega^{2} M_{\text {slosh }}$ (or alternatively, the sloshing mass $M_{\text {slosh }}$ can be considered), natural frequency $\omega$ and damping ratio $\zeta$.

The engine thrust $T$, pivot point position $x_{P V P}$, engine nozzle's dynamics damping ratio $\zeta_{a c t}$ and natural frequency $\omega_{a c t}$ are assumed to be known constants. And finally, the TWD effect is captured through the coupling coefficients $k_{2}$ and $k_{4}-$ with $k_{2}$ and $k_{41}$ known constants:

$$
\begin{aligned}
& -k_{2}=M_{n o z z l e}\left(x_{P V P}-x_{C G, \text { nozzle }}\right) \\
& -k_{4}=-k_{2}\left(x_{C G}-x_{P V P}\right)+J_{n o z z l e}+M_{n o z z l e}\left(x_{P V P}-x_{C G, n o z z l e}\right)^{2}=-k_{2} x_{C G}+k_{41}
\end{aligned}
$$

When the system is recast in the standard first order ordinary differential equation (ODE) form, a pure linear parameter varying (LPV) model ${ }^{10}$ is obtained, with state vector formed by $[y, \theta, s, \beta]$ plus their first order time derivative, and with input the TVC deflection $\beta_{c}$. The system's $A(p), B(p)$, and $C$ matrices are as follows:

$A=\left[\begin{array}{cccccccc}0 & 0 & 0 & -2 \zeta_{a c t} \omega_{a c t} k_{2} M^{-1} & 0 & 0 & M_{\text {slosh }} \omega^{2} M^{-1} & -\left(T+k_{2} \omega_{a c t}^{2}\right) M^{-1} \\ 0 & 0 & 0 & -2 \zeta_{a c t} \omega_{a c t} k_{4} J^{-1} & 0 & 0 & d M_{\text {slosh }} \omega^{2} M^{-1} & \left(T\left(x_{P V P}-x_{C G}\right)-k_{4} \omega_{a c t}^{2}\right) J^{-1} \\ 0 & 0 & -2 \zeta \omega & 2 \zeta_{a c t} \omega_{a c t}\left(k_{2} M^{-1}+k_{4} d J^{-1}\right) & 0 & 0 & -\omega^{2}\left(1+M_{\text {sloss }}\left(M^{-1}+d^{2} J^{-1}\right)\right) & \left(T+k_{2} \omega_{a c t}^{2}\right) M^{-1}-d\left(T\left(x_{P V P}-x_{C G}\right)-k_{4} \omega_{a c t}^{2}\right) J^{-1} \\ 0 & 0 & 0 & -2 \zeta_{a c t} \omega_{a c t} & 0 & 0 & 0 & -\omega_{a c t}^{2} \\ 1 & 0 & 0 & 0 & 0 & 0 & 0 & 0 \\ 0 & 1 & 0 & 0 & 0 & 0 & 0 & 0 \\ 0 & 0 & 1 & 0 & 0 & 0 & 0 & 0 \\ 0 & 0 & 0 & 1 & 0 & 0 & 0 & 0\end{array}\right]$

$$
B=\left[\begin{array}{c}
k_{2} M^{-1} \\
k_{4} J^{-1} \\
-k_{2} M^{-1}-d k_{4} J^{-1} \\
1 \\
0 \\
0 \\
0
\end{array}\right]
$$

The notation $d=x_{C G, s l o s h}{ }^{-} x_{C G}$ in Eq. (3) is introduced to simplify its presentation. Such a model is linear either in the derived parameters or in their inverse. Furthermore, the state dimension can be further reduced from 8 to 6 . Indeed, solving Eq. (3) for $\ddot{y}$ and substituting into Eq. (1) allows elimination of variables $y$ and $\dot{y}$ from the system.

\section{B. Uncertain Parameters}

The open-loop model just presented depends linearly on the derived set $\left(M, J, x_{C G}, x_{C G, \text { slosh }}, M_{\text {slosh }}, \omega, \zeta\right)$. But these derived parameters are complex nonlinear functions, see Eq. (4), of the set of physical parameters referred to as original $\left(m, j, x_{c g}, m_{p l}, J_{p l}, x_{c g, p l}, \lambda\right)$ and some known numerical constants $\left(D_{0}, c_{0}, \Gamma_{0}, \xi\right.$ and $\left.\zeta_{0}\right)$. The first three original parameters correspond respectively to the mass $m$, moment of inertia $j$ and center of gravity position along the centerline $x_{c g}$ for the launch vehicle without payload. The subsequent three original parameters are the equivalent ones for the payload, while the last one is the payload's cylindrical tanks' height over diameter ratio $\lambda$. Finally, the numerical constants are related to payload tank diameter, $\mathrm{x}$-cg sloshing mass constant, gravity acceleration and sloshing mode and damping coefficients. 


$$
\begin{aligned}
& \begin{array}{c}
M=m_{p l}+m ; \\
x_{C G}=\frac{m_{p l} x_{c g, p l}+m x_{c g}}{M} ; \quad x_{C G, s l o s h}=c_{0}+x_{c g, p l}+D_{0}\left(\frac{\lambda}{2}-\frac{1}{\xi} \tanh (\xi \lambda)\right)\left(\frac{m_{p l}}{\lambda}\right)^{1 / 3} ;
\end{array} \\
& J=j+J_{p l}+m_{p l}\left(x_{c g, p l}-x_{C G}\right)^{2} ; \\
& \zeta=\zeta_{0}\left(\frac{\Gamma_{0}}{T} M\right)^{1 / 4} ; \\
& M_{\text {slosh }}=\frac{0.3}{\xi\left(\xi^{2}-1\right)} \frac{m_{p l}}{\lambda} \tanh (2 \xi \lambda) ; \quad \omega^{2}=\frac{2 \xi T}{D_{0}}\left(\frac{\lambda}{m_{p l}}\right)^{1 / 3} \frac{1}{M} \tanh (2 \xi \lambda) ;
\end{aligned}
$$

It is important to note that while the original parameters define a regular, hyper-rectangular space $S_{I}$ (whose bounds are well known), the derived parameters populate an irregular region $S_{2}$ whose bounds must be obtained through careful assessment of the hyper-rectangular space $S_{1}$ and Eq. (4). To simplify the analysis, the irregular region $S_{2}$ is over-bounded obtaining as a result a hyper-rectangular extended space $\mathrm{S}_{2}{ }^{+} \supset \mathrm{S}_{2}$, see Figure 2

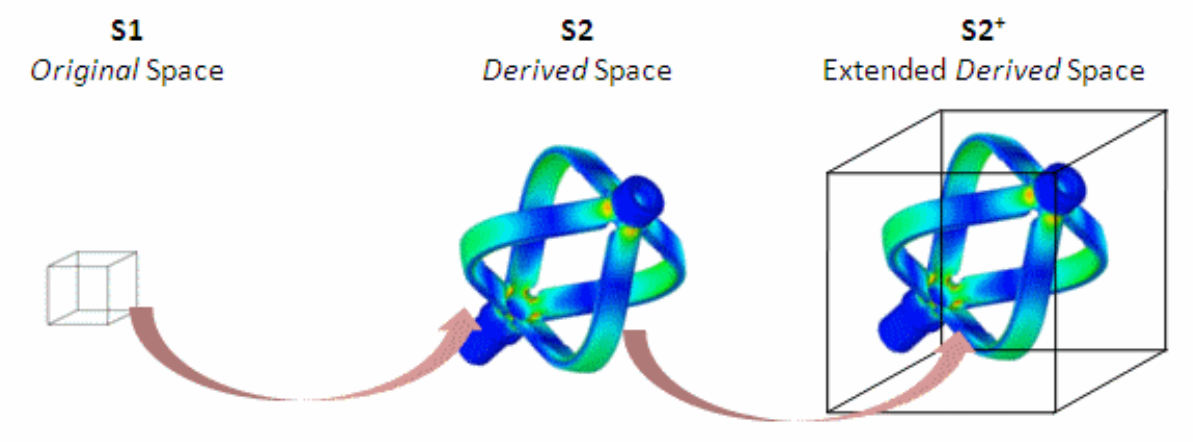

Figure 2 Uncertain parameter spaces: from original to derived to extended derived

\section{Controller}

The digital TVC controller commands a deflection in the thrust vector. The controller follows a PID controller plus structural (notch) filter scheme. The derivative action is implemented via a high-pass second order filter. The notch filter is designed to adequately suppress the elastic-rigid body interactions by properly attenuating all frequency above 1 radians-per-second. Prior to analysis, the digital controller is converted into continuous form using a Tustin transformation.

\section{Analysis Results: Full Payload Case}

In this section robust stability of TVC control law is addressed with respect to the full payload case. That is, we seek to clear the TVC control law for propelling into orbit any generic payload, whose mass, moment of inertia and center of gravity position are inside some specified boundaries.

\section{A. LFT modeling}

As mentioned before, the system of equations can be described in a natural fashion as an LPV system in the derived parameters set $\left(M, J, x_{C G}, x_{C G, \text { slosh }}, M_{\text {slosh }}, \omega, \zeta\right)$, see Eq. (3). The bounds for the derived parameters can be obtained individually based on the nonlinear map $S_{1} \rightarrow S_{2}$, see Eq. (4), but are required to be over-bounded into the extended derived space $\mathrm{S}_{2}{ }^{+}$for analysis purposes. This has the ill effect of "breaking down" the interdependencies among the parameters but simplify the application of the mu analysis algorithm. Attempts to develop an LFT model based on the original parameter set proved impossible due to the complex nonlinearities of Eq. (4).

Using ONERA LFR toolbox ${ }^{9}$, the open-loop derived LFT model description is as follows: 


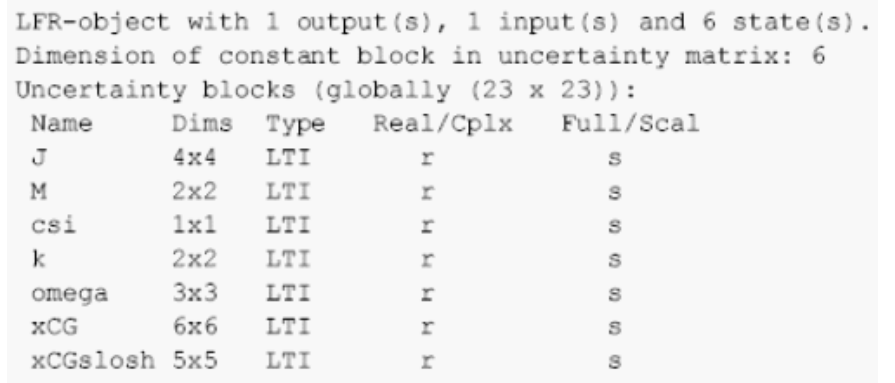

Figure 3 Full payload LFT model based on derived parameter set

Notice that such LPV model is completely general and applicable to the analysis of single payload configurations as well as the full payload domain. Also, it is worthwhile to mention two facts. First, the presence of 6 repetitions of the constant block (related to the way parameter's inversions are dealt with) is automatically resolved when the uncertainty block is normalized. And second, the model is quite compact (total dimension of 23), which makes it suitable for mu analysis.

\section{B. Monte Carlo analysis}

A preliminary stability analysis can be performed via frozen-parameters (i.e. LTI-based) gain and phase margin analysis. The LTI systems can be obtained by direct evaluation of the LPV model of E q. (3) for given values in any of the parameter sets, and gain and phase margins compared to the appointed robustness criteria.

One thousand samples are randomly assigned to each one of the original parameters $\left(m, j, x_{c g}, m_{p l}, J_{p l,} x_{c g, p l}, \lambda\right)$ and the corresponding LTI systems are evaluated after obtaining the appropriate derived parameters values. The LTI systems are found to be consistently stable, with appropriate rigid and flexible gain and phase margins.

Further, a second Monte Carlo using 10,000 samples is performed directly sampling from the extended derived space $\mathrm{S}_{2}{ }^{+}$. This is done to better cross-check the results of mu-analysis performed next as it uses this specific parameter space and the LFT model based on the derived set. Once again, no destabilizing condition is found.

Thus, based on a traditional analysis alone, the TVC control is robust against all payloads, even in the extended derived space.

\section{Mu-analysis: upper-bound}

Application of mu analysis would at first glance appear straightforward based on the format of the LPV/LFT model of Eq. (3). Indeed, the system equations are affine in the derived parameters, such that the resulting LFT model exactly captures the problem dynamics (no simplifications need to be made). Still, it is necessary to bound the derived parameters to facilitate the analysis, thus the extended derived space $\mathrm{S}_{2}{ }^{+}$is used.

Application of mu in this extended derived space means that we are trying to prove robust stability in the face of a much larger system uncertainty than the real one (the hyper-rectangular region upper bounding the derived parameters space is very conservative). Thus, if the mu upper bound ${ }^{6}$ (i.e. guaranteed minimum size of uncertainty for which the system is stable) is lower than 1, then robust stability is guaranteed also for the original space (as it is contained within the extended one) but nothing can be said if the mu upper bound is greater than 1 .

Using the derived LFT model and the extended derived space $\mathrm{S}_{2}{ }^{+}$, the peak of the mu upper bound is greater than one, i.e. the TVC is said to be unstable for the examined parameter set. Albeit an attempt to reduce conservatism by relating the derived parameters among each other (and thus restricting the investigation region in the parameters space) provides better results, it still yields that the system is robust unstable. Furthermore, if this approach is taken, the model complexity (number of parameters' repetition) increases significantly making the analysis much costlier.

\section{Mu-analysis: lower-bound}

Although robust stability for the fully general payload case could not be proved, the use of the associated LFT model is key in proving the advantages of mu to detect worst cases as compared to the traditional Monte Carlo approach. The worst-case can be obtained by looking at the lower bound, which provides the minimum guaranteed size of the uncertainty for which the system is unstable. ${ }^{6}$

Indeed, mu analysis allows us to detect the existence of multiple destabilizing perturbations inside the analyzed parameters space $\mathrm{S}_{2}{ }^{+}$. Recall that no destabilizing perturbation was found using a Monte-Carlo approach in the same LFT model and parameter space, not even after 10,000 samples. Thus, mu has already been capable of determining unstable conditions not picked up by the Monte Carlo approach. Furthermore, existence of multiple destabilizing 
conditions in $\mathrm{S}_{2}{ }^{+}$could be confirmed by Monte Carlo analysis only when the investigation domain was restricted to a small neighborhood (i.e. $10 \%$ variation) centered on the worst case identified by mu. All conditions found by Monte Carlo are very close to the worst case detected by mu.

Since the extended derived space $\mathrm{S}_{2}{ }^{+}$represents only a sufficient condition (i.e. largely over-bounds $\mathrm{S}_{1}$ ) then it is always required to convolve the result from $\mathrm{S}_{2}{ }^{+}$to the original space in order to check whether the result is physically valid and still holding in the latter space. As it turned out, the detected unstable conditions in $\mathrm{S}_{2}{ }^{+}$were not contained in S1 as shown pictorially in Figure 4 so no proof of instability was found for the original set.

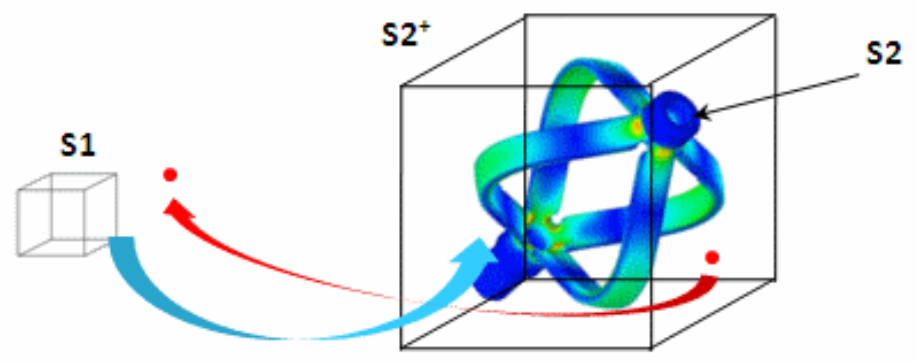

Figure 4 Unstable condition in the extended derived parameter space $S_{2}{ }^{+}$outside the $S_{1}$ parameter space

\section{Analysis Results: Single Payload case}

Analyzing directly the model from Eq. (3) using the extended derived space $S_{2}{ }^{+}$leads to excessive conservatism in the analysis. An alternative approach is to use the knowledge of the payload's mass $m_{p l}$, moment of inertia $J_{p l}$ and center of gravity position $x_{c g, p l}$ to obtain an approximation of Eq. (4) accurate for all the original space $S_{l}$. Note that this approximation can be made without loss of generality, since it is possible to assume that the inertial characteristics of the payload under consideration are quite precisely defined. As a consequence, an LPV model for each desired payload configuration is derived which is linear in the original parameter subset $\left(m, j, x_{c g}\right.$, and $\left.\lambda\right)$. These payload-specific LFT models allow validating the TVC control law in an incremental form, one payload at a time, until the whole payload envelop is analyzed.

The proposed modeling and analysis methodology is tested on nine distinct payloads. These test payloads are chosen such that they span the typical market for a small-size launch vehicle to economically place satellites in the 300 to 2,000 kilograms range into polar and low-Earth orbits.

\section{A. LFT modeling}

When the goal is to analyze the robustness of the TVC control law versus a specified payload, the problem simplifies considerably. To begin with, now it is required to address only a four dimensional original parameter subset $\left(m, j, x_{c g}\right.$, and $\left.\lambda\right)$ since those specific to the payload $\left(m_{p l}, J_{p l}, x_{c g, p l}\right)$ are assigned. This simplification in turn paves the way to tackle the nonlinear parametric dependency from Eq. (4) via a mix of symbolic manipulation and sensible simplification (holding around the point defined by the considered payload).

First, examining Eq. (3) it is noted that it would be advantageous to consider as parameters $M^{-1}$ and $J^{l}$ directly rather than $M$ and $J$, because of the way these parameters appear in the system equations. Next, in order to introduce the original parameter subset each of the equations in the nonlinear map of Eq. (4) are examined in turn. For example, $M^{-1}$ can be directly regarded as an atomic LFT whose parameter's bounds are computed according to $M=m_{P L}+m$ where $m_{P L}$ is assigned once a payload is specified and $m$ is an original parameter thus using $M^{-1}$ is directly connected to using $m$.

The equation for the overall moment of inertia $J$ poses a more serious challenge since an expression is sought on its inverse. Examining the corresponding equation, it is seen that it depends linearly on $j$ and quadratically on the overall center of gravity $x_{C G}$ but due to the available launcher data it can be validated that $J^{1}$ can be quite well approximated based only on the latter. It is therefore easy to find a linear approximation of $J^{I}\left(x_{C G}\right)$ by mean square root, whose accuracy is always better than 2 percent. This approximation must be worked out by inspection of the $\left(x_{C G}, J^{-1}\right)$ plane. An example of such approximation is given in Figure 5 for the first considered payload configuration. In the figure, 10,000 randomly sampled points (blue clouds) are produced by evaluation of the nonlinear transformation mapping the original parameter space into the derived parameter space in order to support such a derivation. The parameter $J^{l}$ approximation on the considered interval is portrayed by the green line. 


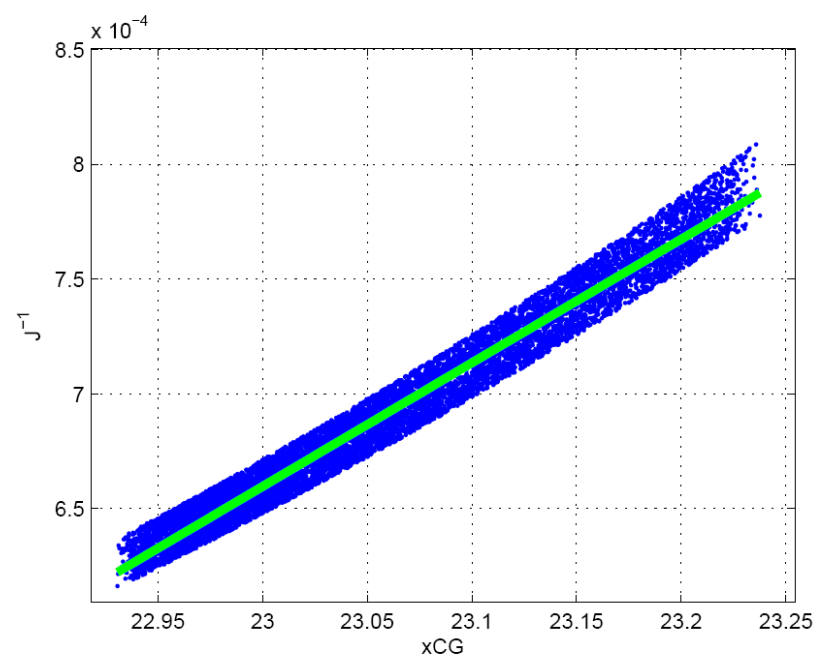

Figure 5 Approximation of $J^{1}$ for a selected payload configuration.

The overall center of gravity $x_{C G}$ can be manipulated to obtain: $x_{C G}=x_{c g}+M^{-1}\left(x_{c g, p k}-x_{c g}\right) m_{p l}$ where $x_{c g}$ is approximated as a constant based on the provided LV data. Note that this approximation is very well justified by the fact that $x_{C G}$ variations are driven mainly by $x_{c g, p l}$ and $M$, which are 2 orders of magnitude bigger than those of $x_{c g}$.

The sloshing mass $M_{\text {slosh }}$ is nonlinear in $\lambda$ through the term $\tanh (2 \xi \lambda)$. Fortunately, for the considered $\lambda$ variation range, this term is bounded by the interval [0.95 - 1]. Thus, assuming a constant $\tanh (2 \xi \lambda)$ in the middle of this interval will cause then less than 2.5 per cent approximation error on $M_{\text {slosh }}$ with linear dependency on $\left(m_{p l} / \lambda\right)$.

The approximation of $\zeta$ relies on the extremely low damping ratios obtained over the whole admissible payload envelope. Although the damping is so light that it can be effectively approximated to zero, doing so would result in an undesired significant mismatch in the system's transfer functions. Thus, a conservative assumption that the damping is equal to the minimum admissible value rather than zero is made. This assumption is conservative as well, since it implies analyzing a more demanding system (by disregarding a potentially energy dissipating contribution). With respect to the approximation of $\omega^{2}$, first note that it is linear in $\lambda^{1 / 3}$ and $M^{-1}$ (after using the above $\tanh (2 \xi \lambda)$ approximation). Further, it is s possible to exploit the fact that $\omega$ is actually used in the equations rather than its square and that its range is such that it can be replaced by a linear approximation on $\omega^{2}$, and thus linearly in $\lambda^{1 / 3}$ and $M^{-1}$.

Finally, the only remaining parameter to approximate is the sloshing center of gravity $x_{C G, s l o s h}$. This term is linear in $\left(m_{p l} / \lambda\right)^{1 / 3}$ and nonlinear through $\lambda 2-\tanh (\xi \lambda) / \xi$. Approximating this nonlinear function is not as trivial as it was for $\tanh (2 \xi \lambda)$ since the variation is much more relevant now. An effective approach is to split the $\lambda$ variation range in two contiguous intervals: $I_{1}=[0.5-1.1]$ and $I_{2}=[1.1-2.0]$. Then, the term $\lambda 2-\tanh (\xi \lambda) / \xi$ can be accurately approximated by two linear relations on $\lambda$, one for each interval.

With the above approximations and simplifications, an LFT model based on $M^{-1}$ and $\lambda^{1 / 3}$ can be obtained:

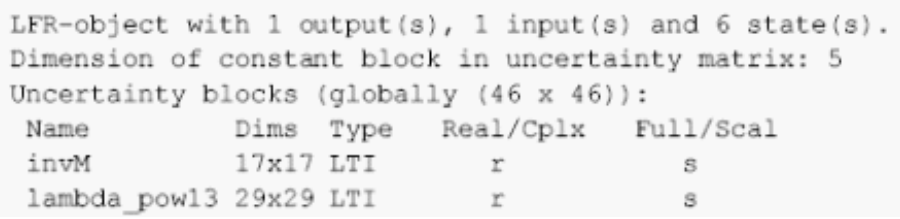

Figure 6 Single payload LFT model based on mixed original and derived parameter set

\section{B. Monte Carlo analysis}

A preliminary stability analysis can be performed via frozen-time parameters (LTI-based) gain and phase margin analysis. The LTI systems can be obtained by direct evaluation of the previous LPV model for a given set of parameters' values, and gain and phase margins compared to the appointed robustness criteria.

For each one of the 9 payloads considered in this study, 1000 samples are randomly assigned to each of the four selected original parameters $\left(m, j, x_{c g}, \lambda\right)$ and the corresponding LTI systems are evaluated. The LTI systems are found to be consistently stable, with appropriate rigid and flexible gain and phase margins. 


\section{Mu-analysis:}

The LFT models obtained for analysis in the single payload case do not allow directly applying mu for clearance of the TVC control for flight, since the analysis space is still too large for being cleared in a single run. In order to circumvent the problem, an approach based on segmentation of the analysis space is used. Thus, the system robust stability is proved by showing that the system is stable on all contiguous subsets $s_{1} \ldots s_{n}$ such that their union $S_{I}=U\left\{s_{1}, \ldots, s_{n}\right\}$ coincides with the complete investigation space.

If the result of these analyses is condensed in a single mu upper bound plot, then the system robust stability on all the investigation domain $S_{1}$ is proven when the peak value of the envelope of upper bound plots obtained on each and every subsets $s_{i}(1 \leq i \leq n)$ is smaller than one.

Overall, $20 \mathrm{mu}$ analyses are required to clear each payload. These 20 analyses arise from the two LFT models obtained from the split of $\lambda^{l / 3}$ into the two regions, $I_{1}$ and $I_{2}$, combined with the split of the parameter $M^{-1}$ into 10 contiguous regions.

The resulting upper bound envelop for one of the considered payloads is shown in Figure 7. It is seen that the TVC control law is thus robustly stable in the original parameter subset. In addition, notice how the mu upper bound is generally quite flat with the exception of the frequency range from 2 to 6 radians per second. Because this frequency band fairly matches the stopband of the TVC structural filter it is possible to draw two conclusions. First, fuel sloshing is potentially the sizing factor in proving robust stability of the TVC laws. Secondly, the frequency overlap testifies the suitability of the notch filter design, which is providing its lowpass action where most needed, i.e. right across the sloshing mode frequency band.

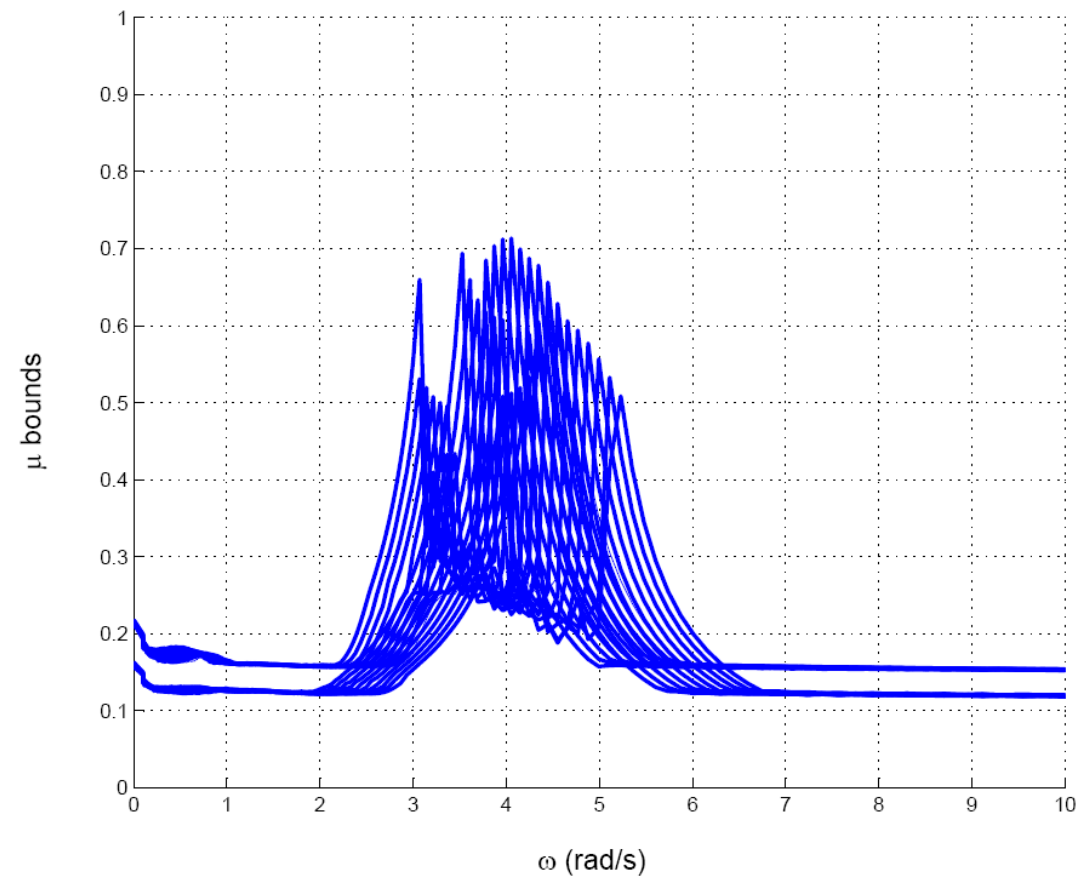

Figure 7 Cumulative mu upper bound proving the TVC robust stability for an assigned payload.

\section{Conclusions}

Within the frame of a European Space Agency (ESA) study entitled "Robust Flight Control System Design Verification and Validation Framework" (RFCS) work is performed with the objective of demonstrating the advantage of using modern analysis techniques through their application to the $\mathrm{V} \& \mathrm{~V}$ of a complex launch vehicle. In this article, an axial-symmetric launch vehicle subject to fuel sloshing was used to exemplify the use of the structured singular value as an analysis tool. It was shown that for the case of the full payload envelope, mu analysis had to be applied in a conservatively large parameter space where it identified worst-cases not picked up by Monte Carlo -although these cases turned out to be outside the physical parameter space of the LV. For the case of a specified payload, mu analysis provided a computational efficient approach to quickly guarantee the robust stability of the vehicle. 


\section{Acknowledgments}

This work is funded by the European Space Agency under ESA-ESTEC contract AO-1-6322/09/NL/JK.

\section{References}

${ }^{1}$ Fielding et al, Advanced Techniques for Clearance of Flight Control Laws. Lecture Notes in Control and Information Science. Berlin: Springer-Verlag, 2002.

${ }^{2}$ Jacklin, S.A., Schumann, J.M., Gupta, P.P., Richard, R., Guenther, K., and Soares, F., "Development of Advanced Verification and Validation Procedures and Tools for the Certification of Learning Systems in Aerospace Applications," Proceedings of Infotech aerospace Conference, Arlington, VA, 2005.

${ }^{3}$ Doyle, J., "Analysis of feedback systems with structured uncertainties," IEE Proceedings Part D, Vol. 129, No. 6, 1982, pp. 242-250.

${ }^{4}$ Packard, A., and Doyle, J.C, "Structural Singular Value with repeated blocks", American Control Conference, Atlanta, 1988.

${ }^{5}$ Packard A., and Doyle, J.C., "Quadratic stability with red and complex perturbations," IEEE Transactions on Automatic Control, Vol. 35, No. 1, 1990.

${ }^{6}$ Balas, G. J., Doyle, J. C., Glover, K., Packard A., Smith, R., The mu analysis and synthesis toolbox. MUSYN and The Mathworks, 1992.

${ }^{7}$ Beck, C., and Doyle, J.C., "Mixed mu upper bound computation," IEEE Conference on Decision and Control, 1992.

${ }^{8}$ Hesham Shageer and Gang Tao, "Modeling and Adaptive Control of Spacecraft with Fuel Slosh: Overview and Case Studies," AIAA Guidance, Navigation, and Control Conference and Exhibit, AIAA 2007-6434, Hilton Head, South Carolina, 2007.

${ }^{9}$ Magni,J.F., "User Manual of the Linear Fractional Representation Toolbox" V2.0, 2006, http://www.onera.fr/staffen/jeanmarc-biannic.

${ }^{10}$ Shamma, J., Cloutier, J., "Gain-Scheduled Missile Autopilot Design Using Linear Parameter Varying Transformations," Journal of Guidance, Control, and Dynamics, Vol. 16, No. 2, 1993, pp. 256-261. 